\title{
BMJ Open High INtensity Interval Training In pATiEnts with intermittent claudication (INITIATE): protocol for a multicentre, proof-of-concept, prospective interventional study
}

\author{
Sean Pymer (D) , Amy Harwood, ${ }^{2,3}$ Said lbeggazene (D) , \\ Gordon McGregor (D) , 2,4,5 Chao Huang, ${ }^{6}$ Maureen Twiddy, ${ }^{6}$ Adam R Nicholls, ${ }^{3}$ \\ Lee Ingle, ${ }^{3}$ Sean Carroll, ${ }^{3}$ Judith Long, ${ }^{1}$ Marjorie Rooms, ${ }^{7}$ I C Chetter, ${ }^{1}$ On behalf of \\ INITIATE investigator group
}

To cite: Pymer S, Harwood A, lbeggazene S, et al. High INtensity Interval Training In pATiEnts with intermittent claudication (INITIATE): protocol for a multicentre, proof-of-concept, prospective interventional study. BMJ Open 2020;10:e038825. doi:10.1136/ bmjopen-2020-038825

- Prepublication history for this paper is available online. To view these files, please visit the journal online (http://dx.doi. org/10.1136/bmjopen-2020 038825).

Received 25 March 2020 Revised 08 April 2020 Accepted 15 April 2020

Check for updates

(C) Author(s) (or their employer(s)) 2020. Re-use permitted under CC BY. Published by BMJ.

For numbered affiliations see end of article.

Correspondence to

Sean Pymer;

sean.pymer@hey.nhs.uk

\section{ABSTRACT}

Introduction The first-line recommended treatment for patients with intermittent claudication (IC) is a supervised exercise programme (SEP), which includes a minimum of 2-hours of exercise per week over a 12-week period. However, provision, uptake and adherence rates for these SEP programmes are poor, with time constraints cited as a common participant barrier. High-intensity interval training (HIIT) is more time-efficient and therefore has the potential to overcome this barrier. However, evidence is lacking for the role of HIIT in those with IC. This proofof-concept study aims to consider the safety, feasibility, tolerability and acceptability of a HIIT programme for patients with IC.

Methods and analysis This multicentre, singlegroup, prospective, interventional feasibility study will recruit 40 patients with IC, who will complete 6 weeks of HIIT, 3 times a week. HIIT will involve a supervised programme of $10 \times 1 \mathrm{~min}$ high-intensity cycling intervals at $85 \%-90 \%$ peak power output (PP0), interspaced with $10 \times 1$ min low intensity intervals at $20 \%-25 \%$ PPO. PPO will be determined from a baseline cardiopulmonary exercise test (CPET) and it is intended that patients will achieve $\geq 85 \%$ of maximum heart rate from CPET, by the end of the second HIIT interval. Primary outcome measures are safety (occurrence of adverse events directly related to the study), programme feasibility (including participant eligibility, recruitment and completion rates) and HIIT tolerability (ability to achieve and maintain the required intensity). Secondary outcomes include patient acceptability, walking distance, CPET cardiorespiratory fitness measures and quality of life outcomes.

Ethics and dissemination Ethical approval was obtained via a local National Health Service research ethics committee (Bradford Leeds - 18/YH/0112) and recruitment began in August 2019 and will be completed in October 2020. Results will be published in peer-reviewed journals and presented at international conferences and are expected to inform a future pilot randomised controlled trial of HIIT versus usual-care SEPs.

Trial registration number NCT04042311; Pre-results.

\section{Strengths and limitations of this study}

This study will assess the safety and feasibility of a novel, pragmatic high-intensity interval training programme for patients with intermittent claudication.

- It will also consider acceptability of the programme via qualitative methods of patient feedback.

- As a limitation, due to the single-group design it is not possible to identify if patients who choose to take part in this study are simply those who would have also chosen to take part in a usual-care exercise programme.

\section{INTRODUCTION}

Peripheral arterial disease (PAD) is caused by atherosclerotic lesions in the arteries supplying the lower limbs, reducing blood flow. ${ }^{1}$ PAD is relatively common, agedependent and increasing in its prevalence. In 2010, it was estimated that PAD affected 202 million people globally, with those aged 75 or older having an approximately eightfold risk compared with those aged less than $60 .^{2}{ }^{3}$ Compounded by population ageing and an increase in the prevalence of diabetes, it was estimated that the number of people living with PAD increased over the previous decade by $13 \%$ and $29 \%$ in high-income and low-middle-income countries, respectively. ${ }^{3}$

Symptomatic PAD typically presents as intermittent claudication (IC), defined as a reproducible ambulatory leg pain, in the calf and/or thigh and/or buttocks, caused by an oxygen supply-demand imbalance, relieved by rest. ${ }^{45}$ As such, IC negatively impacts on walking ability, functional capacity, quality of life (QoL) and daily activities, while also leading to a markedly increased mortality 
risk. ${ }^{6-11}$ The recommended treatment strategy for IC is non-invasive and includes pharmacological risk factor management and exercise therapy, via a supervised exercise programme (SEP). ${ }^{12-14}$ SEPs should consist of a minimum of 2-hours of exercise per week for a 12-week period, with patients encouraged to exercise to the point of maximal pain. ${ }^{12}$ SEPs are supported by high-quality evidence for their clinical and cost-effectiveness, ${ }^{15}$ with evidence also suggesting that SEPs are equal to primary stenting for symptomatic improvement, which is maintained for a year after programme completion. ${ }^{1617}$

Despite the irrefutable evidence for the benefit of SEPs, just $39 \%$ of UK vascular centres provide access to one, ${ }^{18}$ and for those that do, uptake and completion rates are suboptimal. One review demonstrated that only $25 \%$ of screened patients are recruited to a programme, ${ }^{19}$ with time cited as the most common barrier for participation. ${ }^{20}$ Furthermore, the current recommendations for SEPs appear to adopt a 'one size fits all' approach which is not based on any objective measure of functional capacity, potentially limiting physiological and symptomatic benefits. One alternative that is both time-efficient and prescribed based on the gold-standard measure of cardiopulmonary exercise testing (CPET), is highintensity interval training (HIIT). HIIT, therefore, has the potential to overcome the previously cited programmerelated drawbacks of traditional SEP. HIIT has demonstrated similar or superior benefits, when compared with traditionally prescribed exercise, in patients with coronary artery disease, chronic heart failure, hypertension, obesity and metabolic syndrome characteristics. ${ }^{21-24}$ HIIT has been highlighted as a potentially preferred treatment option in those with IC, though the evidence in this population is much more limited. ${ }^{20}$ Initial systematic review evidence has indicated that HIIT has the potential to provide clinical and symptomatic benefits, though there was significant heterogeneity between published studies in terms of HIIT modality, frequency, intensity and duration. ${ }^{25}$ The authors recommended that future appropriately designed studies consider shorter-term and low-volume HIIT programmes for patients with IC.

Therefore, the aim of this multicentre proof-ofconcept study is to consider the safety, tolerability, feasibility and acceptability of a short-term, low-volume HIIT programme in those with IC.

\section{METHODS AND ANALYSIS}

High Intensity Interval Training In pATiEnts With Intermittent Claudication (INITIATE) is a pragmatic, singlegroup, multicentre and prospective interventional proof-of-concept study. The study design and inclusion/ exclusion criteria have been informed by a previous, single-centre study including 30 patients. ${ }^{26}$

For this study, participants will be recruited consecutively and perform 6 weeks of HIIT. Study interventions and outcome assessments will be conducted by research staff that due to the nature of the study cannot be blinded. This protocol adheres to the Standard Protocol Items: Recommendations for Clinical Trials (SPIRIT) guidelines, and we used the SPIRIT checklist when writing this protocol. ${ }^{27} 28$

\section{Setting}

INITIATE will be conducted at two UK centres; 1 . The Academic Vascular Surgical Unit, Hull Royal Infirmary, Kingston-Upon-Hull and 2. Atrium Health, Centre for Exercise and Health, Coventry and Warwickshire Hospitals National Health Service (NHS) Trust, Coventry. Sponsorship is provided by Hull University Teaching Hospitals NHS Trust and funding provided by the National Institute for Health Research, Research for Patient Benefit programme. Recruitment commenced in August 2019, with a recruitment target of 40 patients (20 per site). Recruitment is anticipated to be completed by October 2020.

\section{Study registration}

The study was prospectively registered on ClinicalTrials. gov and the study registration data set is given in table 1 . Any amendments required to this protocol will seek approvals from the research ethics committee and will be outlined (with reasons) in the final published report.

\section{How the sample will be selected}

This study will recruit patients with IC secondary to PAD referred to a usual-care SEP, with a confirmed diagnosis of IC by resting and/or postexercise ankle-brachial pressure index (ABPI) and/or documented significant atherosclerosis on radiological imaging.

\section{Inclusion criteria}

- Aged $\geq 18$ years.

- $\mathrm{ABPI}<0.9$ at rest or a systolic pressure drop of $\geq 20 \mathrm{~mm}$ $\mathrm{Hg}$ at the ankle after exercise testing.

- Ability to walk unaided.

- English speaking and able to comply with exercise instructions.

\section{Exclusion criteria}

- Unable to provide informed consent.

- Critical limb threatening ischaemia/rest pain/tissue loss.

- Active cancer treatment.

- Significant comorbidities precluding safe participation in exercise testing and/or training according to the American College of Sports Medicine (ACSM) guidelines. $^{29}$

- Resting/uncontrolled tachycardia (>100 bpm) and/ or resting/uncontrolled hypertension (systolic blood pressure $>180 \mathrm{~mm} \mathrm{Hg}$ or diastolic blood pressure $>100 \mathrm{~mm} \mathrm{Hg}$ ).

- Symptomatic hypotension.

Additional exclusion criteria

Following baseline CPET, patients will be withdrawn and prevented from continuing their involvement in the study if there is any evidence of: 
Table 1 Study registration items

\begin{tabular}{ll}
\hline Data category & Information \\
\hline $\begin{array}{l}\text { Primary registry and } \\
\text { identifying number }\end{array}$ & ClinicalTrials.gov NCT04042311 (Workstream 2) \\
$\begin{array}{l}\text { Date of registration in } \\
\text { primary registry }\end{array}$ & $01 / 08 / 2019$
\end{tabular}

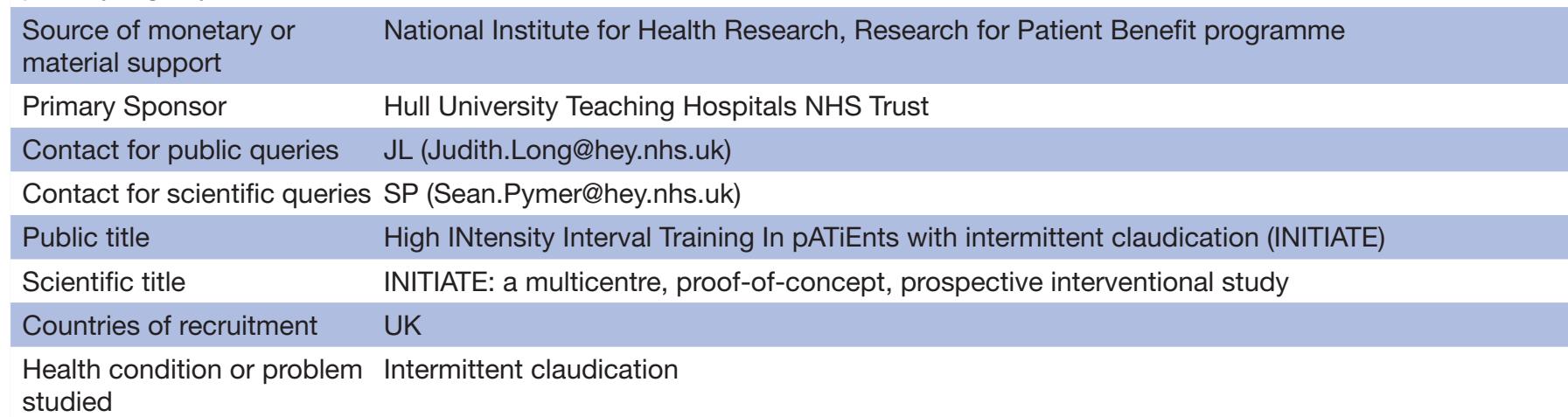

Intervention High-intensity interval training

Key inclusion and exclusion Ages eligible for the study: $\geq 18$ years

criteria Sexes eligible for the study: all

Accepts healthy volunteers: no

Inclusion criteria:

Community dwelling adults aged 18 or over.

$\mathrm{ABPI}<0.9$ at rest or a drop of more than $20 \mathrm{~mm} \mathrm{Hg}$ after exercise testing

Ability to walk unaided

English speaking and able to comply with exercise instructions

Exclusion Criteria:

Unable to provide informed consent

Critical limb threatening ischaemia/rest pain/tissue loss

Active cancer treatment

Significant comorbidities precluding safe participation in exercise testing and / or training according to the American College of Sports Medicine guidelines ${ }^{28}$

Resting/uncontrolled tachycardia (>100 bpm) and/or resting/uncontrolled hypertension (systolic blood pressure $>180 \mathrm{~mm} \mathrm{Hg}$ or diastolic blood pressure $>100 \mathrm{~mm} \mathrm{Hg}$ )

Symptomatic hypotension

$\begin{array}{ll}\text { Additional exclusion criteria: } & \begin{array}{l}\text { Exercise-induced myocardial ischaemia or significant haemodynamic compromise (manifesting } \\ \text { as anginal symptoms, significant ECG changes or an abnormal blood pressure response). }\end{array} \\ \text { Study type } & \begin{array}{l}\text { Interventional } \\ \text { Allocation: single group assignment } \\ \text { Primary purpose: Treatment }\end{array} \\ & 12 / 08 / 2019 \\ \text { Date of first enrolment: } & 40 \text { patients } \\ \text { Target sample size: } & \text { Recruiting } \\ \text { Recruitment status: } & \text { Safety: occurrence of adverse and serious adverse events } \\ \text { Primary outcomes: } & \text { Feasibility: eligibility, recruitment and completion rates } \\ & \text { Tolerability: assessing reasons for withdrawal, and identifying ability to reach and maintain the } \\ & \text { required intensity. } \\ \text { Acceptability: patient feedback via semistructured interview } \\ \text { Efficacy: pain-free and maximal walking distance } \\ \text { Quality of life } \\ \text { Cardiorespiratory measures } \\ \text { Ankle brachial pressure index }\end{array}$

ABPI, Ankle-Brachial Pressure Index. 
- Exercise-induced myocardial ischaemia or significant haemodynamic compromise (manifesting as anginal symptoms, significant ECG changes or an abnormal blood pressure response).

\section{Study procedures}

The participant pathway for the study is shown in figure 1 . Briefly, patients who are deemed eligible for a usual SEP will be referred to the research team and their medical history reviewed to determine potential eligibility for INITIATE. Those appearing to meet the eligibility criteria will be sent an invitation letter and patient information sheet. Patients will then be contacted at least a week later via telephone to give them the opportunity to ask any questions and confirm if they are willing to participate. Those who decide to participate will be asked to attend a baseline visit where eligibility will be confirmed before informed consent is obtained. Those who decline the study will be offered SEP as per usual care.

Acceptability of the study and intervention will be assessed using qualitative interviews. The participant information sheet and consent form will include a clause that outlines the conduction of an interview with a subset of patients. The interview is optional, and participants can decline to be interviewed. Baseline and follow-up procedures will include a full and detailed medical history, medication and symptom review, assessment of ABPI and a Gardner-Skinner graded treadmill test, ${ }^{30}$ followed by postexercise ABPI. For those who are confirmed eligible, spirometry and CPET will be subsequently undertaken. QoL measures will also be collected using the Medical Outcomes Study Short-Form 36 (SF-36) and the Kings College Hospitals Vascular QoL (VascuQoL) Questionnaires, both of which have demonstrated good reliability and validity in this patient population. ${ }^{31} 32$ Following baseline CPET, exercise ECG and haemodynamic response will be evaluated to reassess eligibility to undergo HIIT. Those who exhibit exercise-induced ischaemia or an abnormal haemodynamic response to volitional exhaustion will be withdrawn from the study and referred back to the vascular consultant/required specialty as appropriate. Given CPET is not part of routine care, all patients will sign informed consent prior to undergoing it. Measurements will be taken before starting the programme (baseline/week 0), immediately after completing the programme (week 6), then 12 weeks later (week 18). A further follow-up will be conducted 4 weeks (week 10) after programme completion at the Hull site only.

\section{Intervention}

This study will adapt a pragmatic and flexible HIIT protocol, based on a similar protocol currently being investigated in those with coronary artery disease. ${ }^{33}$ Patients will attend three HIIT sessions per week for a period of 6 weeks, totalling 18 sessions. If participants miss sessions, the intervention period can be extended for up to two additional weeks to allow these sessions to be completed. Those not completing 18 sessions over the extended 8-week period will be deemed to have satisfactorily completed the intervention as long as they have undertaken $>80 \%$ of the HIIT sessions (ie, $\geq 15$ out of 18 sessions). All patients completing the allotted 6-8 weeks for the intervention (regardless of whether they have completed $\geq$ or $<15$ sessions) will be followed up. Those selecting to discontinue the intervention prematurely will be withdrawn, but the information collected up to their withdrawal will be retained and may still be used.

The intervention will be performed using a cycle ergometer (Wattbike Trainer, Wattbike, Nottingham, UK), with exercise prescription based on the peak workload achieved during the cycle CPET at baseline. Variations from high to low intensity cycling will be achieved by altering the cycle cadence (rpm). Although walking is often the recommended mode of exercise for those with IC and a treadmill based HIIT programme has been previously considered, ${ }^{34} 35$ a cycle was chosen for the current investigation for a number of reasons. First, the use of a treadmill may preclude patients from reaching their prescribed HIIT training zones due to limiting claudication pain. Stationary cycling may also reduce the risk of falls, given the balance limitation often experienced by patients with IC. ${ }^{36}$ In addition, it has been demonstrated that the limiting symptoms during treadmill walking are often experienced in the leg, predominantly the calf, whereas the limiting symptoms during cycling are much more varied. ${ }^{37}$ Finally, it has also been noted that cycle testing is better tolerated than treadmill testing in those with IC, which is important considering that the HIIT training zone requires the patient to exercise intermittently to near-peak exertion levels. ${ }^{38}$

Our HIIT work to rest ratio will be 1:1 (1 min highintensity work interspaced with $1 \mathrm{~min}$ of low-intensity work), with patients completing 10 intervals for an overall exercise session time of $20 \mathrm{~min}$. If required, a titrated introduction to the HIIT programme will be used with fewer exercise intervals being completed in the first 2 weeks. Patients will also be allowed to complete less than 10 intervals for longer than the first 2 weeks if required but will be encouraged to complete 10 as soon as possible thereafter. HIIT workloads will be set at $85 \%-90 \%$ of the peak power output achieved during the baseline CPET. Application of this workload aims to achieve $85 \%-100 \%$ peak heart rate (HRpeak) from CPET by the end of the second interval. Our personal experience with cardiac patients has demonstrated that patients may exceed their HRpeak (from baseline CPET) during HIIT sessions. This is also likely to be the case for those with IC, especially those who are unable to achieve a maximal effort CPET. We will adopt a pragmatic approach to this by allowing it to occur without adjusting workload but will monitor on a case-bycase basis and reduce cycling intensity when it is deemed appropriate. We will also record these occurrences to allow appropriate reporting. All sessions will be preceded and followed by a 10 min warm-up and cool-down as is standard practice for exercise rehabilitation for older adults with chronic disease. 
Patient seen by vascular consultant, diagnosis of IC confirmed and referred for exercise programme.

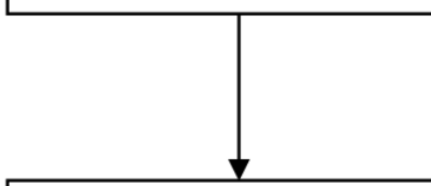

Patient is sent patient information sheet detailing the 6-week HIIT exercise intervention and will be called at least a week later

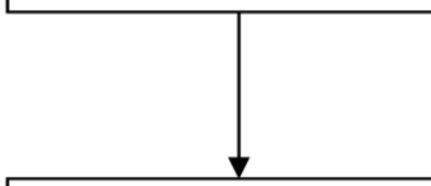

Patient chooses to partake in research and baseline assessment arranged.
Patient declines to partake in the research. Is offered usual 12-week SEP without research. If this is declined, they will be referred back to their consultant for future management.

Baseline assessment -

eligibility confirmed, informed

consent obtained, and

outcome measures assessed

Immediate post-intervention follow-up - outcome measures reassessed, and semistructured interview conducted (if appropriate)

12-week follow-up - outcome measures reassessed, and patient exits study and are referred back to their consultant for management.

Figure 1 Participant study flow. HIIT, high-intensity interval training; IC, intermittent claudication; SEP, supervised exercise programme 


\section{Outcome measures}

\section{Primary outcomes}

The primary outcomes for this study are safety, feasibility and tolerability.

Safety will be assessed by determining the occurrence of any adverse or serious adverse events related to the intervention or study procedures. These events will be recorded in accordance with the Good Clinical Practice (GCP) decision tree for adverse event reporting and where applicable events will be reported to the sponsor and/or research ethics committee.

Feasibility will be assessed by considering eligibility ( $n=$ eligible/screened), recruitment ( $n=$ recruited/eligible) and adherence ( $n=$ completed/recruited). As such, the number of patients screened, recruited, commencing and completing (either satisfactorily or unsatisfactorily) the HIIT programme will be monitored at each site.

Tolerability will be assessed by considering reasons for withdrawal (ie, if they are related to the intervention) and identifying the number of patients able to reach and maintain the required intensity (ie, $\geq 85 \%$ HRMax by the end of the second interval) for the full 10 intervals. Tolerability will also assess whether patients can complete the full 10 intervals by the end of the second week.

\section{Secondary outcomes}

Secondary outcome measures include, acceptability, pain-free and maximal walking distance, ABPI, QoL and cardiorespiratory measures, collected during CPET.

\section{Acceptability}

will be assessed by conducting semistructured interviews at both sites using a sample of patients in three groups:

Group 1: Patients who are eligible for the study but decide not to participate (non-consenters). The interviews will explore reasons why patients chose not to participate in the study and whether study material could be amended to be more appealing. As these patients have declined participation in the study, they will sign an interview specific consent form.

Group 2: Those who agree to participate in, and complete, the exercise programme (completers). The interviews will explore patient's experiences of the HIIT programme, how acceptable they found it, whether they enjoyed it and whether they would be willing to undertake it again. They will also be asked to provide information related to potential barriers to participation in the programme and study, and any changes they may feel are required.

Group 3: Those who agree to participate but discontinued after at least one session (withdrawals). Patients will be asked about their reasons for discontinuation and what, if anything, could have been modified to prevent withdrawal from the study.

An interview topic guide with a predetermined set of open questions will be used but the interviews will be flexible to allow the interviewer to ask further probing questions based on patient responses, and for patients to raise issues not explicitly covered by the topic guide. All interviews will be audio recorded using a Dictaphone, transcribed verbatim and anonymised.

\section{Pain-free and maximal walking distance}

Pain-free and maximal walking distance will be determined using the Gardner/Skinner treadmill test which starts at $2.0 \mathrm{mp} /$ hour and $0 \%$ gradient, with gradient increasing by $2 \%$ every $2 \mathrm{~min}$, while the speed remains constant, up to a maximum of $15 \mathrm{~min}$. For those unable to walk on the treadmill at $2.0 \mathrm{mp} /$ hour the speed will be reduced, but this speed will remain consistent at all follow-up visits to ensure standardisation. Patients will indicate when they begin to feel IC pain, which will be recorded as pain-free walking distance and the patient will continue until the pain is too severe and they need to stop, which will be recorded as maximal walking distance. Patients able to walk for $15 \mathrm{~min}$ will be excluded.

\section{Quality of life}

QoL will be assessed with both a generic and disease specific questionnaire. The SF-36 will be used as it is recommended as the most appropriate generic tool for those with lower limb ischaemia. ${ }^{39}$ The SF-36 gives a scoring profile across eight domains, ranging from 0 to 100 , with 0 indicating worst possible health and 100 best possible health. Scales can also be combined to create a physical and mental component summary.

The disease-specific questionnaire will be the VascuQoL which was designed for use in studies involving patients with lower limb ischaemia. It contains 25 items subdivided into 5 domains, which are rated on a 7-point scale with 1 representing the worst score and 7 the best. A sum score is also calculated by dividing the total score by 25 .

\section{Cardiorespiratory measures}

Cardiorespiratory function will be assessed at each time point using an individualised ramp based cycle CPET, conducted in accordance with international guidelines. ${ }^{40}{ }^{41}$ Patients will be screened for contraindications to CPET and continuously monitored for indications for termination as per the ACSM guidelines. ${ }^{29}$ The CPET will be preceded by a 3 min period of rest on the bike to obtain resting measurements followed by a 3 min reference period of unloaded cycling followed by a progressive individualised ramp protocol designed to elicit volitional exhaustion within 8-12 min, concluding with a recovery period. ${ }^{41}$ Patients will be encouraged to maintain 65-70 rpm throughout the test until they are limited by volitional fatigue. Monitoring will be via 12-lead ECG, blood pressure, oxygen saturation and rating of perceived exertion (RPE). Attainment of a maximal effort will be considered if the patient achieves 2 out of the following three criteria; achieving $\geq 85 \%$ age-predicted maximum heart rate, a respiratory exchange ratio $>1.10$ and an RPE $>17 .{ }^{42}$ However, based on a previous study, $\sim 25 \%$ of patients with IC are unable to achieve this, meaning it will not be applied as an exclusion and patients will continue 
in the study, regardless of whether it is achieved. ${ }^{26}$ Breathby-Breath gas analysis will be conducted (MedGraphics Ultima2 Medgraphics, St Paul, Minnesota, USA or Ergostick, LoveMedical, Manchester, UK) to allow determination of a number of cardiorespiratory fitness parameters.

\section{Ankle-Brachial Pressure Index}

The systolic blood pressure will be measured bilaterally in the brachial, dorsalis pedis and posterial tibial arteries using a hand-held doppler and appropriately sized sphygmomanometer, with ABPI determined by dividing the higher ankle pressure of each leg with the highest arm pressure. Patients will be deemed eligible if they have an ABPI of $<0.9$ or a postexercise systolic blood pressure drop at the ankle of $\geq 20 \mathrm{~mm} \mathrm{Hg}$.

\section{Sample size}

As this feasibility proof-of-concept study does not aim to make any statistical comparison nor estimate an SD for future power calculations, there is no formal sample size requirement. We aim to recruit 20 patients from each site over the recruitment period, for a total of 40 patients.

\section{Data collection and management}

Data will be collected by the study team across $3 / 4$ time points dependant on site. Data will be collected continuously for the qualitative study, based on the time at which patients decline, withdraw from or complete the intervention, until the point of data saturation. Data will be collected and retained in accordance with the General Data Protection Regulation (2018). All patients will be given a study code to ensure anonymity. Data will be stored via paper case report forms (CRFs) in codesecured research offices at the vascular laboratory in Hull Royal Infirmary and Coventry and Warwickshire University Hospital respectively with the same identification code. These CRFs will be periodically scanned and sent to the team at Hull Royal Infirmary, who will manage the electronic and physical database, via email with end-to-end encryption. This database will be stored on a computer in the code-secured research office that is password protected and has both antivirus and firewall software. Only authorised members of the research team will have access to the patient data and transfer of data will be via Trust encrypted, password-protected remote storage devices or secure nhs.net mail. Only authorised members of the research team will have access to the final dataset which will be stored for 5 years following study completion.

\section{Data analysis}

Where applicability allows, the study will be reported in accordance with the Consolidated Standards of Reporting Trials (2010) statement extension to pilot and feasibility studies. ${ }^{43}$ Descriptive statistics will be reported for our feasibility, tolerability and safety (proof-of-concept) outcomes. Descriptive statistics for our secondary outcomes will be reported to inform potential future studies in terms of clinical and QoL outcome measures.
The qualitative data will be analysed using an inductive thematic analysis, whereby themes are identified from within the data. ${ }^{44}$ The researcher will read and reread the transcripts to identify patterns of responses within the data that are related to the research question and can be grouped together under a theme heading. The approach will be inductive, which means that the themes are data driven, thus emerging from the data, and do not fit into a pre-existing coding frame. ${ }^{44}$

\section{Patient and public involvement}

The background patient and public involvement (PPI) work for this study was supported by a grant from the NIHR Research Design Service Yorkshire and the Humber. Consequently, two focus group sessions, each involving five patients with a confirmed diagnosis of IC and experience of undertaking a standard SEP, were conducted which informed the design of this study. In addition, this PPI group is committed to continuous contribution during the research study, with the chair of the PPI group invited to attend all trial steering committee meetings. We also aim to hold 3-4 PPI meetings over the course of the study to aid with addressing potential recruitment or retention issues and aid with dissemination of the study findings.

\section{ETHICS AND DISSEMINATION}

Protocol approval was obtained via a local NHS research ethics committee (Bradford Leeds - 18/YH/0112) and all patients will provide informed consent prior to participation, which will be obtained by study personnel with appropriate GCP training.

On completion, study results will be published in peerreviewed journals and presented at international scientific meetings. In addition, with our PPI group, we will disseminate findings to the public, which will include lay summaries to participants and vascular charities such as the Circulation Foundation (Registered Charity Number: 1102769). The expected impact for this study is the development of a new time-efficient exercise programme for patients with IC, which is more acceptable, thus improving uptake and adherence. Should this study support the feasibility of HIIT for patients with IC, we aim to undertake a multicentre, pilot randomised controlled trial comparing HIIT to standard SEPs, which can inform a definitive trial, which has potential to impact on international guidelines.

\section{Author affiliations}

${ }^{1}$ Academic Vascular Surgical Unit, Hull York Medical School, Hull, UK

${ }^{2}$ Centre for Sport, Exercise and Life Sciences, Coventry University, Coventry, New South Wales, UK

${ }^{3}$ Department of Sport, Health and Exercise Science, University of Hull, Hull, UK ${ }^{4}$ Department of Cardiac Rehabilitation, Centre for Exercise and Health, University Hospitals Coventry and Warwickshire NHS Trust, Coventry, UK

${ }^{5}$ Warwick Clinical Trials Unit, Warwick Medical School, University of Warwick, Coventry, UK

${ }^{6}$ Institute of Clinical and Applied Health Research, Hull York Medical School, University of Hull, Hull, UK 
${ }^{7}$ Hull, UK

Twitter Amy Harwood @amyharwood91 and Gordon McGregor @HIITorMISSUK Collaborators INITIATE study group.

Contributors SP, LI, ICC and AH conceived the study and initiated study design. $\mathrm{SP}, \mathrm{AH}, \mathrm{SI}, \mathrm{GM}, \mathrm{CH}, \mathrm{MT}, \mathrm{ARN}, \mathrm{LI}, \mathrm{SC}, \mathrm{JL}, \mathrm{MR}$ and ICC contributed fully to the study design, and will contribute to ongoing data collection and provide input during study management and steering committee meetings and day-to-day running of the study, when required. SP, LI, ICC, AH, ARN, CH, MT, MR, GM, and SI are grant holders. SP, AH, SI, GM, CH, MT, ARN, LI, SC, JL, MR and ICC contributed to the refinement of the protocol and approved the final manuscript.

Funding This work is supported by National Institute for Health Research, Research for Patient Benefit programme grant number PG-PB041820014.

Competing interests None declared.

Patient and public involvement Patients and/or the public were involved in the design, or conduct, or reporting, or dissemination plans of this research. Refer to the Methods section for further details.

Patient consent for publication Not required.

Ethics approval (Bradford Leeds -18/YH/0112). IRAS ID: 237067.

Provenance and peer review Not commissioned; peer reviewed for ethical and funding approval prior to submission.

Open access This is an open access article distributed in accordance with the Creative Commons Attribution 4.0 Unported (CC BY 4.0) license, which permits others to copy, redistribute, remix, transform and build upon this work for any purpose, provided the original work is properly cited, a link to the licence is given, and indication of whether changes were made. See: https://creativecommons.org/ licenses/by/4.0/

ORCID iDs

Sean Pymer http://orcid.org/0000-0003-1685-2091

Said Ibeggazene http://orcid.org/0000-0001-9457-7887

Gordon McGregor http://orcid.org/0000-0001-8963-9107

\section{REFERENCES}

1 Hiatt WR. Medical treatment of peripheral arterial disease and claudication. N Engl J Med 2001;344:1608-21.

2 Criqui $\mathrm{MH}$, Fronek A, Barrett-Connor E, et al. The prevalence of peripheral arterial disease in a defined population. Circulation 1985;71:510-5.

3 Fowkes FGR, Rudan D, Rudan I, et al. Comparison of global estimates of prevalence and risk factors for peripheral artery disease in 2000 and 2010: a systematic review and analysis. Lancet 2013;382:1329-40.

4 Beebe HG. Intermittent claudication: effective medical management of a common circulatory problem. Am J Cardiol 2001;87:14-18.

5 Morley RL, Sharma A, Horsch AD, et al. Peripheral artery disease. BMJ 2018;360:j5842.

6 Criqui $\mathrm{MH}$, Langer RD, Fronek $\mathrm{A}$, et al. Mortality over a period of 10 years in patients with peripheral arterial disease. $N$ Engl J Med 1992;326:381-6.

7 Pell JP. Impact of intermittent claudication on quality of life. The Scottish vascular audit group. Eur J Vasc Endovasc Surg 1995:9:469-72.

8 Meru AV, Mittra S, Thyagarajan B, et al. Intermittent claudication: an overview. Atherosclerosis 2006;187:221-37.

9 Golomb BA, Dang TT, Criqui MH. Peripheral arterial disease: morbidity and mortality implications. Circulation 2006;114:688-99.

10 Norgren L, Hiatt WR, Dormandy JA, et al. Inter-society consensus for the management of peripheral arterial disease (TASC II). J Vasc Surg 2007;45 Suppl S:S5-67.

11 Criqui $\mathrm{MH}$, Aboyans V. Epidemiology of peripheral artery disease. Circ Res 2015;116:1509-26.

12 NICE. Peripheral arterial disease: diagnosis and management. Clinical guidance 147, 2012.

13 Aboyans V, Ricco J-B, Bartelink M-LE, et al. 2017 ESC guidelines on the diagnosis and treatment of peripheral arterial diseases, in collaboration with the European Society for vascular surgery (ESVS) document covering atherosclerotic disease of extracranial carotid and vertebral, mesenteric, renal, upper and lower extremity arteries endorsed by: the European stroke organization (ESO) the task force for the diagnosis and treatment of peripheral arterial diseases of the European Society of cardiology (ESC) and of the European Society for vascular surgery (ESVS). Eur Heart $J$ 2017;39:763-816.

14 Gerhard-Herman MD, Gornik HL, Barrett C, et al. 2016 AHA/ACC guideline on the management of patients with lower extremity peripheral artery disease: Executive summary: a report of the American College of Cardiology/American heart association Task force on clinical practice guidelines. J Am Coll Cardiol 2017;69:1465-508

15 Lane R, Harwood A, Watson L, et al. Exercise for intermittent claudication. Cochrane Database Syst Rev 2017;12:CD000990.

16 Murphy TP, Cutlip DE, Regensteiner JG, et al. Supervised exercise versus primary stenting for claudication resulting from aortoiliac peripheral artery disease: six-month outcomes from the claudication: exercise versus endoluminal revascularization (clever) study. Circulation 2012;125:130-9.

17 Murphy TP, Cutlip DE, Regensteiner JG, et al. Supervised exercise, stent revascularization, or medical therapy for claudication due to aortoiliac peripheral artery disease: the clever study. J Am Coll Cardiol 2015;65:999-1009.

18 Harwood AE, Smith GE, Broadbent E, et al. Access to supervised exercise services for peripheral vascular disease patients. The Bulletin of the Royal College of Surgeons of England 2017;99:207-11.

19 Harwood A-E, Smith GE, Cayton T, et al. A systematic review of the uptake and adherence rates to supervised exercise programs in patients with intermittent claudication. Ann Vasc Surg 2016;34:280-9.

20 Harwood AE, Hitchman LH, Ingle L, et al. Preferred exercise modalities in patients with intermittent claudication. $J$ Vasc Nurs 2018;36:81-4.

21 Hannan AL, Hing W, Simas V, et al. High-Intensity interval training versus moderate-intensity continuous training within cardiac rehabilitation: a systematic review and meta-analysis. Open Access $J$ Sports Med 2018;9:1-17.

22 Liou K, Ho S, Fildes J, et al. High intensity interval versus moderate intensity continuous training in patients with coronary artery disease: a meta-analysis of physiological and clinical parameters. Heart Lung Circ 2016;25:166-74.

23 Elliott AD, Rajopadhyaya K, Bentley DJ, et al. Interval training versus continuous exercise in patients with coronary artery disease: a metaanalysis. Heart Lung Circ 2015;24:149-57.

24 Weston KS, Wisløff U, Coombes JS. High-Intensity interval training in patients with lifestyle-induced cardiometabolic disease: a systematic review and meta-analysis. Br J Sports Med 2014;48:1227-34.

25 Pymer S, Palmer J, Harwood AE, et al. A systematic review of highintensity interval training as an exercise intervention for intermittent claudication. J Vasc Surg 2019;70:2076-87.

26 Pymer S, Ibeggazene S, Palmer J, et al. A prospective observational cohort study considering the feasibility and tolerability of high intensity interval training as a novel treatment therapy for patients with intermittent claudication. medRxiv 2020.

27 Chan A-W, Tetzlaff JM, Gøtzsche PC, et al. Spirit 2013 explanation and elaboration: guidance for protocols of clinical trials. $B M J$ 2013;346:e7586.

28 Chan A-W, Tetzlaff JM, Altman DG, et al. Spirit 2013 statement: defining standard protocol items for clinical trials. Ann Intern Med 2013;158:200-7.

29 ACSM. ACSM's guidelines for exercise testing and prescription. 9th ed. Baltimore: Lippincott, Williams, \& Wilkins, 2014.

30 Gardner AW, Skinner JS, Cantwell BW, et al. Progressive vs singlestage treadmill tests for evaluation of claudication. Med Sci Sports Exerc 1991;23:402???408-8.

31 Morgan MB, Crayford T, Murrin B, et al. Developing the vascular quality of life questionnaire: a new disease-specific quality of life measure for use in lower limb ischemia. J Vasc Surg 2001;33:679-87.

32 Brazier JE, Harper R, Jones NM, et al. Validating the SF-36 health survey questionnaire: new outcome measure for primary care. BMJ 1992;305:160-4.

33 McGregor G, Nichols S, Hamborg T, et al. High-Intensity interval training versus moderate-intensity steady-state training in UK cardiac rehabilitation programmes (HIIT or miss UK): study protocol for a multicentre randomised controlled trial and economic evaluation. BMJ Open 2016;6:e012843.

34 Gardner AW, Montgomery PS, Flinn WR, et al. The effect of exercise intensity on the response to exercise rehabilitation in patients with intermittent claudication. J Vasc Surg 2005;42:702-9.

35 Tew GA, Harwood AE, Ingle L, et al. The bases expert statement on exercise training for people with intermittent claudication due to peripheral arterial disease. The Sport and Exercise Scientist 2018. 
36 Gohil RA, Mockford KA, Mazari F, et al. Balance impairment, physical ability, and its link with disease severity in patients with intermittent claudication. Ann Vasc Surg 2013;27:68-74.

37 Askew CD, Green S, Hou XY, et al. Physiological and symptomatic responses to cycling and walking in intermittent claudication. Clin Physiol Funct Imaging 2002;22:348-55.

38 Tuner SL, Easton C, Wilson J, et al. Cardiopulmonary responses to treadmill and cycle ergometry exercise in patients with peripheral vascular disease. J Vasc Surg 2008;47:123-30.

39 Chetter IC, Spark JI, Dolan P, et al. Quality of life analysis in patients with lower limb ischaemia: suggestions for European standardisation. Eur J Vasc Endovasc Surg 1997;13:597-604.
40 Balady GJ, Arena R, Sietsema K, et al. Clinician's guide to cardiopulmonary exercise testing in adults: a scientific statement from the American heart association. Circulation 2010;122:191-225.

41 Fletcher GF, Ades PA, Kligfield P, et al. Exercise standards for testing and training: a scientific statement from the American heart association. Circulation 2013;128:873-934.

42 Nichols S, Taylor C, Ingle L. A clinician's guide to cardiopulmonary exercise testing 2: test interpretation. Br J Hosp Med 2015;76:281-9.

43 Eldridge SM, Chan CL, Campbell MJ, et al. Consort 2010 statement: extension to randomised pilot and feasibility trials. BMJ 2016;355:i5239.

44 Braun V, Clarke V. Using thematic analysis in psychology. Qual Res Psychol 2006;3:77-101. 